Rapid Reviews COVID-19•

\title{
Review 2: "Anti-PF4 levels \\ of patients with VITT do \\ not reduce 4 months \\ following AZD1222 \\ vaccination"
}

Helmuth Haslacher ${ }^{1}$, Thomas Perkmann ${ }^{1}$

${ }^{1}$ Medical University of Vienna

Published on: Sep 14, 2021

License: Creative Commons Attribution 4.0 International License (CC-BY 4.0). 


\section{$\underline{\text { RR:C19 Evidence Scale rating by reviewer: }}$}

- Potentially informative. The main claims made are not strongly justified by the methods and data but may yield some insight. The results and conclusions of the study may resemble those from the hypothetical ideal study, but there is substantial room for doubt. Decision-makers should consider this evidence only with a thorough understanding of its weaknesses, alongside other evidence and theory. Decisionmakers should not consider this actionable, unless the weaknesses are clearly understood and there is other theory and evidence to further support it.

$* * * * * * * * * * * * * * * * * * * * * * * * * * * * * * * * * * * * * * *$

\section{Review:}

The ongoing SARS-CoV-2 pandemic is one of our generation's most challenging medical, economic, and humanitarian crises. The rapid development of vaccines against the disease has allowed a slow return to normality in some countries. The protective effect, especially against severe courses, is estimated to be comparably high. In addition to the novel mRNA vaccines, vector vaccines were also used and were cheaper and more stable than the former. However, serious side effects were recorded in sporadic cases, which became known as VITT (vaccine-induced thrombotic thrombocytopenia). In many countries, however, the benefits of the vaccines have been prioritized over the potential risks. The clinical picture of VITT is similar to heparininduced thrombocytopenia (HIT) and is diagnostically characterized by high levels of PF4 (platelet factor 4) antibodies in PF4-antibody-ELISAs. Patients sometimes develop extensive thrombosis at atypical sites, and the mortality rate is high. Therapy includes anticoagulation with DOAKs. However, it is still unclear when DOAK treatment should be discontinued.

Nicolson et al. aimed to contribute to a better understanding of the clinical course of VITT by presenting data from seven patients with VITT beyond hospital discharge. In their pre-print article, "Anti-PF4 levels of patients with VITT do not reduce 4 months after AZD1222 vaccination," the authors describe PF4 antibody levels along with results of functional platelet activation tests at the time of diagnosis, as well as for up to 5 months afterward. The authors concluded that while PF4 antibody levels remained high, there was a marked decrease in the ability of the patient's serum to activate platelets. Although the cause of this observation remains unclear, the authors speculate on the more rapid decrease of a possible co-factor, which may still be 
unknown and could explain the discrepancy between PF4 antibody levels and functional test results.

The manuscript has several strengths. The manuscript includes the finding that platelets treated with the patient's serum could be activated in the presence of PF4 even months after hospital discharge, from which therapeutic indications could be derived. However, the manuscript also has relevant limitations. For example, the article's main conclusion is not adequately supported. The authors use a qualitative test to estimate PF4 antibody concentration. The measured ODs thus represent only an orienting value and must not be seen as a reliable "antibody titer" (see Table 2). Furthermore, the test was developed to diagnose heparin-induced thrombocytopenia with an OD of 0.400 as a threshold for positivity. However, samples from patients with VITT usually present extraordinarily high ODs in these assays, which was also confirmed by the authors' data. Therefore, it can be assumed that these are all above the linear measurement range. A reliable quantification is therefore extremely unlikely. At the time of the follow-up, the measured values (except for the patient in whom antibody production was suppressed by rituximab administration) only slightly decreased or remained similarly high. However, due to the utmost semi-quantitative nature of such an assay, the dimension of the change cannot be determined. This aspect still needs to be considered in the relatively brief discussion.

In summary, we agree with the authors that PF4 antibodies are still detectable in patients with VITT several months after diagnosis. At the same time, the functional activity of these antibodies seems to decrease in the follow-up samples. However, the authors should consider that these findings could be merely a result of the different discriminatory capacities of the binding and functional assay. In addition, the PF4 antibody tests used, which were developed for HIT diagnostics, could be outside the range in which VITT antibody levels are detected in a roughly quantitative and linear manner. 